Publ. RIMS, Kyoto Univ. Ser. A

Vol. 3 (1967), pp. 129-137

\title{
Un théorème d'existence pour une équation aux dérivées partielles parabolique non linéaire, II
}

\author{
Par
}

Masuo Hukuhara

\section{Introduction.}

Dans notre article précédent [5], nous avons traité l'équation parabolique non linéaire

$$
\partial^{2} y / \partial x^{2}=g(t, x, y, \partial y / \partial t) .
$$

Nous voulons maintenant étendre notre résultat au cas de l'équation suivante

$$
\partial^{2} y / \partial x^{2}=g(t, x, y, \partial y / \partial x, \partial y / \partial t),
$$

dont le second membre dépend de $\partial y / \partial x$. La méthode s'appuie sur un théorème d'existence dû à M. Nagumo [4], dont l'énoncé voici.

Considérons l'équation différentielle ordinaire $d u$ second ordre

$$
d^{2} y / d x^{2}=g(x, y, d y / d x),
$$

dont le second membre est continu dans le domaine fermé

$$
\alpha \leqq x \leqq \alpha^{\prime}, \quad \underline{\omega}(x) \leqq y \leqq \bar{\omega}(x), \quad \underline{\Omega}(x, y) \leqq y^{\prime} \leqq \bar{\Omega}(x, y) ;
$$

$\underline{\omega}(x)$ et $\bar{\omega}(x)$ sont des fonctions $\in \mathrm{C}^{2}\left[\alpha, \alpha^{\prime}\right]$ telles que l'on ait

$$
\begin{aligned}
& \underline{\omega}\left(\alpha^{\prime}\right)=\bar{\omega}(\alpha)=\beta, \quad \underline{\omega}\left(\alpha^{\prime}\right) \leqq \beta^{\prime} \leqq \bar{\omega}\left(\alpha^{\prime}\right), \\
& \underline{\omega}^{\prime \prime}(x) \geqq g\left(x, \underline{\omega}(x), \underline{\omega}^{\prime}(x)\right), \\
& \bar{\omega}^{\prime \prime}(x) \leqq g\left(x, \bar{\omega}(x), \bar{\omega}^{\prime}(x)\right) ;
\end{aligned}
$$

$\underline{\Omega}(x, y)$ et $\bar{\Omega}(x, y)$ sont des fonctions appartenant $\grave{a}$

$$
C^{1}\left\{(x, y) ; \alpha \leqq x \leqq \alpha^{\prime}, \underline{\omega}(x) \leqq y \leqq \bar{\omega}(x)\right\}
$$

Received May 8, 1967. 
et satisfaisant aux inégalités

$$
\underline{\Omega}(\alpha, \beta) \leqq \underline{\omega}^{\prime}(\alpha) \leqq \bar{\omega}^{\prime}(\alpha) \leqq \bar{\Omega}(\alpha, \beta), \underline{\Omega}(x, y) \leqq \bar{\Omega}(x, y),
$$

(1.6) $\quad g(x, y, \underline{\Omega}(x, y))-\underline{\Omega_{x}}(x, y)-\underline{\Omega}_{y}(x, y) \underline{\Omega}(x, y)>0$,

$$
g(x, y, \bar{\Omega}(x, y))-\bar{\Omega}_{x}(x, y)-\bar{\Omega}_{y}(x, y) \bar{\Omega}(x, y)<0 .
$$

Sous ces hypothèses il existe au moins une solution de l'équation (1.2) satisfaisant aux conditions suivantes:

$$
\begin{aligned}
& y(\alpha)=\beta, \quad y\left(\alpha^{\prime}\right)=\beta^{\prime}, \\
& \underline{\omega}(x) \leqq y(x) \leqq \bar{\omega}(x), \\
& \underline{\Omega}(x, y(x)) \leqq y^{\prime}(x) \leqq \bar{\Omega}(x, y(x)) .
\end{aligned}
$$

Remarque. Comme nous avons remarqué dans l'article précédent, $\bar{\omega}^{\prime}(x)$ peut admettre la discontinuité de première espèce. Si elle est discontinue en $\xi$, il suffit de supposer

$$
\bar{\omega}^{\prime}(\xi-0)>\bar{\omega}^{\prime}(\xi+0) .
$$

$\bar{\omega}^{\prime \prime}(x)$ aussi peut admettre la discontinuité de première espèce. Si elle est discontinue en $\xi$, il suffit de supposer que $\bar{\omega}^{\prime \prime}(\xi \pm 0)$ sont au plus égales au second membre de $\overline{(1.4)}$, où $x$ est remplacé par $\xi$.

Il en est de même de $\underline{\omega}(x)$.

\section{Enonce du théorème d'existence.}

Nous supposons que la fonction $g\left(t, x, y, y^{\prime}, z\right)$ est continue et admet les dérivées partielles $g_{t}, g_{y}, g_{y^{\prime}}, g_{z}$ satisfaisant aux inégalités

$$
\begin{gathered}
\left|g_{t}\left(t, x, y, y^{\prime}, z\right)\right| \leqq A, \\
\left|g_{y}\left(t, x, y, y^{\prime}, z\right)\right| \leqq B, \\
\left|g_{y^{\prime}}\left(t, x, y, y^{\prime}, z\right)\right| \leqq B^{\prime}, \\
0<\lambda \leqq g_{z}\left(t, x, y, y^{\prime}, z\right) \leqq \mu, \\
\left|g_{w}\left(t, x, y, y^{\prime}, z\right)-g_{w}\left(\bar{t}, x, \bar{y}, \bar{y}^{\prime}, \bar{z}\right)\right| \\
\leqq H|t-\bar{t}|+K|y-\bar{y}|+K^{\prime}\left|y^{\prime}-\bar{y}^{\prime}\right|+L|z-\bar{z}|
\end{gathered}
$$


dans le domaine fermé

$$
0 \leqq t \leqq T_{0}, \quad 0 \leqq x \leqq 1, \quad|y| \leqq \beta, \quad\left|y^{\prime}\right| \leqq \beta^{\prime}, \quad|z| \leqq \gamma,
$$

où $w$ représente l'une quelconque des variables $t, y, y^{\prime}, z$.

Sous ces hypothèses, si $T\left(\leqq T_{0}\right)$ est un nombre positif assez petit, l'équation (1.1) admet au moins une solution définie dans un domaine fermé

$$
0 \leqq t \leqq T, \quad 0 \leqq x \leqq 1
$$

et telle que

$$
y(0, x)=0, \quad y(t, 0)=0, \quad y(t, 1)=0 .
$$

Si l'on considère (1.1) comme équation qui définit $\partial y / \partial t$ en fonction de $t, x, y, \partial y / \partial x, \partial^{2} y / \partial x^{2}$, on obtient une fonction uniforme à cause des inégalités $(2.1)_{z}$. Nous pouvons donc écrire l'équation donnée sous la forme

$$
\partial y / \partial t=f\left(t, x, y, \partial y / \partial x, \partial^{2} y / \partial x^{2}\right) .
$$

\section{Définition des fonctions $y_{n}, z_{n}, u_{n}$.}

Nous définissons successivement les fonctions $y_{n}, z_{n}, u_{n}$ comme dans l'article précédent, c'est-à-dire par les formules suivantes

$$
\left\{\begin{array}{l}
t_{n}=n h, \quad T=N h \\
y_{0}=o ; \\
y_{n}(0)=y_{n}(1)=0: \\
z_{n}=\frac{y_{n}-y_{n-1}}{h}=f\left(t_{n}, x, y_{n}, y_{n}^{\prime}, y_{n}^{\prime \prime}\right) \\
u_{n}=\frac{z_{n}-z_{n-1}}{h}
\end{array}\right.
$$

La valeur de $T$ sera déterminée au $\mathrm{n}^{\circ} 6$. Nous désignons par $P_{N}$ la fonction polygonale dont les sommets sont $\left(t_{n}, y_{n}\right)$, c'est-à-dire nous posons

$$
P_{N}(t)=\frac{\left(t-t_{n-1}\right) y_{n}+\left(t_{n}-t\right) y_{n-1}}{h}
$$

peur $t_{n-1} \leqq t \leqq t_{n}$. On a alors 


$$
D^{+} P_{N}(t)=z_{n}
$$

pour $t_{n-1} \leqq t<t_{n}$.

Nous appliquerons le théorème d'existence de M. Nagumo à l'équation en $z=z_{n}$ qui s'écrit

$$
\begin{aligned}
h z^{\prime \prime}= & g\left(t_{n}, x, y_{n-1}+h z, y_{n-1}^{\prime}+h z^{\prime}, z\right) \\
& -g\left(t_{n-1}, x, y_{n-1}, y_{n-1}^{\prime}, z_{n-1}\right) .
\end{aligned}
$$

\section{Limitation des $z_{n}$.}

La condition à laquelle doit satisfaire la fonction

$$
\bar{\omega}(x)=z_{n-1}+h u
$$

pour qu'elle soit majorante pour l'équation (3. 3), s'écrit

$$
\begin{aligned}
h^{2} u^{\prime \prime} \leqq & g\left(t_{n}, x, y_{n-1}+h z_{n-1}+h^{2} u, y_{n-1}^{\prime}+h z_{n-1}^{\prime}+h^{2} u^{\prime}, z_{n-1}+h u\right) \\
& -2 g\left(t_{n-1}, x, y_{n-1}, y_{n-1}^{\prime}, z_{n-1}\right)+g\left(t_{n-2}, x, y_{n-2}, y_{n-2}^{\prime}, z_{n-2}\right) .
\end{aligned}
$$

Si $u>u_{n-1}$, le second membre est au moins égal à

$$
\begin{aligned}
& -h^{2}\left(H+K\left\|z_{n-1}\right\|+K^{\prime}\left\|z_{n-1}^{\prime}\right\|+L\left\|u_{n-1}\right\|\right)\left(1+\left\|z_{n-1}\right\|+\left\|z_{n-1}^{\prime}\right\|+\left\|u_{n-1}\right\|\right) \\
& -B h^{2} \cdot u-B^{\prime} h^{2}\left|u^{\prime}\right|+\lambda h\left(u-u_{n-1}\right) .
\end{aligned}
$$

Si nous supposons

$$
\left\|z_{n-1}\right\| \leqq c_{n-1}, \quad\left\|z_{n-1}^{\prime}\right\| \leqq C^{\prime}, \quad\left\|u_{n-1}\right\| \leqq d_{n-1},
$$

la condition en $u$ est remplie lorsque

$$
\begin{aligned}
h u^{\prime \prime} \leqq & -h\left(1+C^{\prime}+c_{n-1}+d_{n-1}\right)\left(H+K^{\prime} C^{\prime}+K c_{n-1}+L d_{n-1}\right) \\
& -B h \cdot u-B^{\prime} h\left|u^{\prime}\right|+\lambda\left(u-u_{n-1}\right) .
\end{aligned}
$$

Cette condition est aussi suffisante pour que la fonction

$$
\underline{\omega}(x) \equiv z_{n-1}-u
$$

soit minorante pour l'équation (3.3). On aura donc

$$
\left|z_{n}-z_{n-1}\right| \leqq u
$$

Posons 


$$
u=\left\{\begin{array}{lll}
a_{n} x-b x^{2} & \text { pour } & 0 \leqq x \leqq \delta_{n} \\
d_{n} & \text { pour } & \delta_{n} \leqq x \leqq 1
\end{array}\right.
$$

ce qui exige que l'on ait

$$
\begin{array}{lll}
a_{n} \delta_{n}-b \delta_{n}^{2}=d_{n} & \text { ou } & a_{n}=b \delta_{n}+d_{n} / \delta_{n}, \\
a_{n}-2 b \delta_{n} \geqq 0 & \text { ou } & a_{n} \geqq 2 b \delta_{n} .
\end{array}
$$

Ces deux conditions sont remplies si l'on prend

$$
a_{n}=\sqrt{2 b d_{n}}, \quad \delta_{n}=\sqrt{d_{n} / b} .
$$

La condition (4.2) devient pour $\delta_{n} \leqq x \leqq 1$

$$
\begin{aligned}
& \lambda\left(d_{n}-d_{n-1}\right) \\
\geqq & h\left(1+C^{\prime}+c_{n-1}+d_{n-1}\right)\left(H+K^{\prime} C^{\prime}+K c_{n-1}+L d_{n-1}\right)+B h d_{n} .
\end{aligned}
$$

Cette condition est remplie lorsque

(4.5) $\quad d_{n}=d_{n-1}$

$$
+h \cdot \frac{\left(1+C^{\prime}+c_{n-1}+d_{n-1}\right)\left(H+K^{\prime} C^{\prime}+K c_{n-1}+L d_{n-1}\right)+B d_{n-1}}{\lambda-B h} .
$$

Posons ensuite

$$
c_{n}=c_{n-1}+h d_{n} .
$$

La croissance de la suite $\left\{d_{n}\right\}$ implique celle des suites $\left\{a_{n}\right\} .\left\{c_{n}\right\}$ et $\left\{\boldsymbol{\delta}_{n}\right\}$.

La condition (4.2) est remplie pour $0 \leqq x \leqq \delta_{n}$ lorsque

$$
2 b \geqq\left(1+C^{\prime}+c_{n-1}+d_{n-1}\right)\left(H+K^{\prime} C^{\prime}+K c_{n-1}+L d_{n-1}\right)+B d_{n}+B^{\prime} a_{n} .
$$

Si nous supposons

$$
c_{n-1} \leqq C, \quad d_{n-1} \leqq D
$$

il suffit que l'on ait

$$
\begin{aligned}
2 b \geqq & \left(1+C+C^{\prime}+D\right)\left(H+K C+K^{\prime} C^{\prime}+L D\right) \\
& +B D+2 B^{\prime} \sqrt{b D} .
\end{aligned}
$$

La fonction $u$ est alors complètement déterminée et on a l'inégalité (4.3), qui implique

$$
\left\|z_{n}-z_{n-1}\right\| \leqq h d_{n}
$$




\section{Limitation de $z_{n}^{\prime}$.}

Nous prenons pour $\bar{\Omega}=\varphi(x)$ une fonction ne dépendant que de $x$. La condition ( $\overline{1.6}$ ) peut alors s'écrire

$$
\begin{aligned}
h \varphi^{\prime}(x) & >g\left(t_{n}, x, y_{n-1}+h z, y_{n-1}^{\prime}+h \varphi(x), z\right) \\
& -g\left(t_{n-1}, x, y_{n-1}, y_{n-1}^{\prime}, z_{n-1}\right),
\end{aligned}
$$

où $z$ parcourt l'intervalle

$$
\left|z-z_{n-1}\right| \leqq d_{n}
$$

On peut prendre pour $\varphi$ la solution de l'équation différentielle

$$
\varphi^{\prime}(x)=B^{\prime} \varphi(x)+A+B C+\mu D
$$

avec la condition initiale

$$
\varphi(0)=2 \sqrt{b D}\left(>a_{n}\right) .
$$

On obtient ainsi pour $\bar{\Omega}$ l'expression

$$
\varphi(x)=\frac{A+B C+\mu D}{B^{\prime}}\left(e^{B^{\prime} x}-1\right)+2 \sqrt{b D} \cdot e^{B^{\prime} x} .
$$

On verra dans la suite que l'on peut supposer

$$
\begin{aligned}
C^{\prime} & \geqq \varphi(1) \\
& =\frac{A+B C+\mu D}{B^{\prime}}\left(e^{B^{\prime}}-1\right)+2 \sqrt{b D} \cdot e^{B^{\prime}} .
\end{aligned}
$$

On aura alors

$$
\left|z_{n}^{\prime}\right| \leqq C^{\prime}
$$

\section{Légitimité des évaluations de $z_{n}$.}

Prenons une valeur positive $C^{\prime}$ plus grande que $A / B^{\prime}$ et puis une valeur positive $b$ telle que l'on ait

$$
2 b>\left(1+C^{\prime}\right)\left(H+K^{\prime} C^{\prime}\right) .
$$

Si l'on prend ensuite les constantes assez petites $C$ et $D$, on aura les inégalités (5.2) et (4.8).

Déterminons successivement les valeurs $c_{n}$ et $d_{n}$ par les formules (4.5) et (4.6) avec les valeurs initiales $c_{0}=d_{0}=0$ et puis les valeurs 
$b_{n}$ et $b_{n}^{\prime}$ par

$$
b_{n}=b_{n-1}+h c_{n}, \quad b_{n}^{\prime}=b_{n-1}^{\prime}+h C_{n}^{\prime},
$$

avec les valeurs initiales $b_{0}=b_{0}^{\prime}=0$. Désignons par $Y_{N}(t), Z_{N}(t), U_{N}(t)$ les fonctions polygonales dont les sommets sont $\left(n h, b_{n}\right),\left(n h, c_{n}\right),(n h$, $d_{n}$ ) respectivement. Les points $\left(n h, b_{n}^{\prime}\right)$ se trouvent sur la droite qui est le graphique de la fonction linéaire $t C^{\prime}$. Si l'on fait $N \rightarrow \infty$, les fonctions $X_{N}(t), Z_{N}(t), U_{N}(t)$ convergent respectivement vers les fonctions $Y(t), Z(t), U(t)$ qui constituent la solution du système différentiel

$$
\left\{\begin{array}{l}
\frac{d Y}{d t}=Z, \quad \frac{d Z}{d t}=U \\
\frac{d U}{d t}=\frac{1}{\lambda}\left\{\left(1+C^{\prime}+Z+U\right)\left(H+K^{\prime} C^{\prime}+K Z+L U\right)+B U\right\} \\
Y(0)=Z(0)=U(0)=0 .
\end{array}\right.
$$

La convergence est uniforme dans un intervalle compact où $Y(t)$, $Z(t), U(t)$ sont définies.

Prenons une valeur positive $T\left(\leqq T_{0}\right)$ telle que

$$
\left\{\begin{array}{l}
Y(T)<\beta, T C^{\prime}<\beta^{\prime}, U(T)<D, \\
Z(T)<\min \left\{C^{\prime}, \gamma\right\} .
\end{array}\right.
$$

Si alors $N$ est assez grand, les fonctions $Y_{N}(t), Z_{N}(t), U_{N}(t)$ sont définies certainement dans l'intervalle $0 \leqq t \leqq T$ et on a

$$
\left\{\begin{array}{l}
0 \leqq Y_{N}(t) \leqq \beta, \quad 0 \leqq U_{N}(t) \leqq D, \\
0 \leqq Z_{N}(t) \leqq \min \left\{r, C^{\prime}\right\},
\end{array}\right.
$$

pour $0 \leqq t \leqq T$.

Les inégalités

$$
\left|y_{k}\right| \leqq b_{k},
$$

$$
\left|y_{k}^{\prime}\right| \leqq k h C^{\prime},
$$

$$
\left|z_{k}\right| \leqq c_{k},
$$

$\left|z_{k}^{\prime}\right| \leqq C^{\prime}$,

$$
\left|u_{k}\right| \leqq d_{k}
$$


sont évidemment satisfaites pour $k=0$. Supposons donc qu'elles soient remplies pour $k=n-1<N$. Puisque $u$ définie au $\mathrm{n}^{\circ} 4$ est non négative et ne surpasse pas $d_{n}$, l'inégalité $\left|z-z_{n-1}\right| \leqq u$ implique

$$
\left\{\begin{array}{l}
|z| \leqq c_{n}=Z_{N}(n h) \leqq \gamma \\
\left|y_{n-1}+h z\right| \leqq b_{n}=Y_{N}(n h) \leqq \beta
\end{array}\right.
$$

et on voit que $z_{n-1}+u$ est une fonction majorante pour (3.3) tandis que $z_{n-1}-u$ est une fonction minorante pour (3.3).

Grâce à l'inégalité (5.2), la fonction $\varphi(x)$ définie par (5.1) ne surpasse pas $C^{\prime}$. Puisque l'on a

$$
d_{n}=U_{N}(n h) \leqq D
$$

et

$$
\left|y_{n-1}^{\prime}+h \varphi(x)\right| \leqq b_{n-1}^{\prime}+h C^{\prime}=n h C^{\prime} \leqq \beta^{\prime}
$$

les fonctions

$$
\underline{\Omega}(x)=-\varphi(x), \quad \bar{\Omega}(x)=\varphi(x)
$$

satisfait aux conditions de M. Nagumo relatives à $\underline{\Omega}$ et $\bar{\Omega}$. Le théorème d'existence cité au $\mathrm{n}^{\circ} 2$ est donc applicable et les inégalités (6.5) subsistent pour $k=n$.

Par conséquent les fonctions $y_{k}, z_{k}, u_{k}$ sont certainement définies pour $k=0,1, \cdots, N$ et on a les inégalités (6.5).

\section{Parachèvement de la démonstration du théorème d'existence.}

Comme nous avons expliqué dans notre article précédent, il suffit, pour comléter la démonstration du théorème d'existence, de montrer la compacité des suites $\left\{P_{N}\right\}$ et $\left\{D^{+} P_{N}\right\}$, et pour démontrer la compacité des suites, il suffit de montrer l'équicontinuité des suites et la compacité des suite en chaque valeur de la variable indépendante $t$.

Puisque l'on a (3.2), (6.5) z et $c_{k} \leqq C$, la suite $\left\{P_{N}\right\}$ est équicontinue. Puisque l'on a $(6.5)_{y},(6.5)_{y^{\prime}}$ et $b_{k} \leqq \beta, k h C^{\prime} \leqq T C^{\prime}$, la suite $\left\{P_{N}(t)\right\}$ est compacte si l'on fixe la valeur de $t$. Il en résulte la compacité de la suite de fonctions $\left\{P_{N}\right\}$.

Puisque l'on a (3.2), (6.5),$(6.5)_{z^{\prime}}$ et $c_{k} \leqq \gamma$ la suite $\left\{D^{+} P_{N}(t)\right\}$ 
est comacte si l'on fixe la valeur de $t$. Puisque l'on a (6.5) ${ }_{u}$ et $d_{k} \leqq D$, on a $\left|u_{k}\right| \leqq D$, et la dernière des relation (3.1) montre l'équi-continuité de la suite de fonctions $\left\{D^{+} P_{N}\right\}$.

La démonstration du théorème d'existence est donc complètement achevée.

\section{BIBLIOGRAPHIE}

M. Hukuhara,

[1] Cauchy no Oresen ni yoru Kai no Sonzai Syômei, Kansû Hôteisiki 14 (1961), 3-10.

[2] Le problème aux limites pour un système de deux équations différentiells ordinaires, J. Math. Soc. Japan. 3 (1951), 99-103.

[3] Une propriété de l'application $f\left(x, y, y^{\prime}, \cdots, y^{(n)}\right)$, Funkcial. Ekvac. 5 (1963), 135-144.

[4] La propriété de Kneser globale et le problème aux limites, Publ. RIMS, Kyoto Univ. Ser. A 1 (1966), 129-148.

[5] Un théorème d'existence pour une équation aux dérivées partielles parabolique non linéaire, Japanese J. Math. 36 (1967), 57-66.

T. Kato,

[1] Integration of the equation of evolution in a Banach space, J. Math. Soc. Japan 5 (1953), 208-234.

M. Nagumo,

[1] Über die Differentialgleichung $y^{\prime \prime}=f\left(x, y, y^{\prime}\right)$, Proc. Phys.-Math. Soc. Japan 19 (1937), 129-148.

[2] Über das Randwertproblem der nicht linearen Differentialgleichungen zweiter Ordnung, Ibid. 24 (1942), 845-851.

[3] Eine Art der Randwertaufgabe von Systemen Gewöhnlicher Differentialgleichungen, I, II, Ibid. 25 (1943), 221-226, 384-390.

[4] Dai-2-kai Zyôbibunhôteisiki no Kyôkaiti Mondai, I, II, Kansû Hôteisiki, No. 5 (1939), 27-34; No. 6 (1939), 37-44.

[5] $y^{\prime \prime}=f\left(x, y, y^{\prime}\right)$ no Kyôkaiti Mondai ni tuite, I, II, Ibid., No. 30 (1941), 36-46; No. 31 (1942), 50-52.

H. Okamura,

[1] $y^{\prime \prime}=f\left(x, y, y^{\prime}\right)$ ni tuite, I, II, III, Kansû Hôteisiki, No. 27 (1941), 27-35; No. 30 (1941), 14-19; No. 31 (1942), 32-40. 
